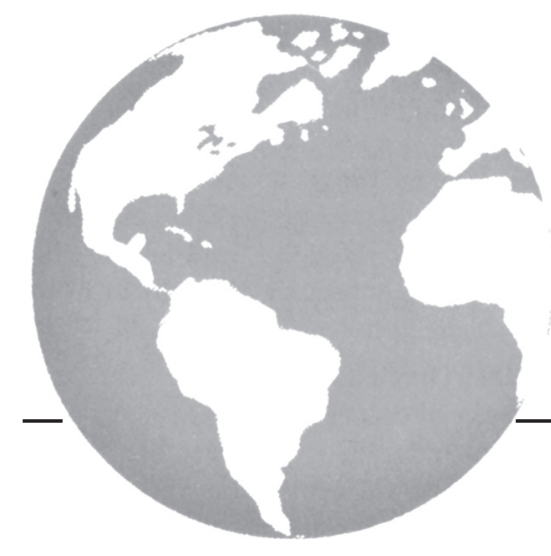

\title{
Extramammary Paget disease: review of patients seen in a non-melanoma skin cancer clinic
}

\author{
J. Pang, * D. Assaad MD, 'ं D. Breen RT(T),,+ J. Fialkov $M D,{ }^{\S}$ \\ O. Antonyshyn MD, ${ }^{\S}$ J. Balogh MD MSc, * M. Tsao MD, * \\ J. Kamra MD, * G. Czarnota MD PhD, ${ }^{*}$ and E.A. Barnes $M{ }^{*}$
}

\begin{abstract}
Extramammary Paget disease (EMPD) is a rare skin disease commonly found in the anogenital region. In this study, we aimed to identify EMPD patients seen in the non-melanoma skin cancer clinic at Odette Cancer Centre and to describe the treatments delivered and outcomes achieved. From 2000 to 2009, 14 patients were seen. Initial treatment recommendations included imiquimod and surgical excision, although half the patients required more than one treatment modality, highlighting the difficulty of achieving complete eradication of this disease.
\end{abstract}

\section{KEY WORDS}

Extramammary Paget disease

\section{INTRODUCTION}

In 1889, Crocker reported the first case of extramammary Paget disease (EMPD) ${ }^{1}$. The lesions Crocker observed had histologic features similar to those of mammary Paget disease, but were located on the perineum. In both diseases, there is intraepithelial infiltration by neoplastic cells, but the origin of EMPD is not certain ${ }^{2}$. The primary or cutaneous form is thought to originate from intraepidermal cells or apocrine glands. The secondary form is associated with an underlying adenocarcinoma, and the skin lesions are thought to represent epidermotropic metastases. However, because only about $35 \%$ or fewer EMPD patients have coexisting malignancies, this later explanation is not consistently applicable ${ }^{3}$.

Extramammary Paget disease is rare; a populationbased study in the Netherlands found an incidence of 0.11 per 100,000 person-years ${ }^{3}$. Extramammary Paget disease is commonly found in the anogenital region, but it can develop on any skin or mucosal surface, particularly in areas rich in apocrine glands, such as the axilla. It usually presents as a slowly expanding, sharply demarcated erythematous or leucoplakic plaque that can be eczematous, crusting, scaling, or (occasionally) ulcerated. Pruritus is the most common presenting symptom, but pain, burning, irritation, and bleeding can also occur ${ }^{2}$. An accurate diagnosis is often delayed by years because of the nonspecific clinical appearance ${ }^{3}$. Treatment options include wide local excision, radiotherapy, or topical therapy, including with imiquimod ${ }^{4}$.

At a multidisciplinary non-melanoma skin cancer (NMSC) clinic held weekly at the Odette Cancer Center, patients are jointly assessed by the dermatology, plastic surgery, and radiation oncology service. Approximately 500 patients are seen annually. The purpose of the present study was to identify patients with EMPD seen in the NMSC clinic and to describe the treatments delivered and outcomes achieved.

\section{PATIENTS AND METHODS}

A retrospective chart review and a search of the NMSC database identified patients with a pathologic diagnosis of EMPD seen in the NMSC clinic between January 2000 and January 2009. Data collected included patient age, sex, tumour site and size, treatment recommendations, and outcomes. Follow-up usually occurred every 3-6 months for up to 5 years. All patients returning for at least 1 follow-up visit were included in the analysis. At each follow-up visit, the presence or absence of disease was recorded, together with any current treatment.

\section{RESULTS}

The review and search identified 14 patients (12 men, 2 women) with 15 EMPD lesions. Mean age at clinic visit was 73 years (range: 58-91 years). Of these 14 patients, 11 were investigated for a possible underlying malignancy (using computed tomography scan of the chest, abdomen, pelvis, and cystoscopy, colonoscopy, and prostate-specific antigen). Prostate cancer was identified in 1 person. A history of prostate cancer was present in 2 patients before their EMPD diagnosis.

The delay from symptom onset to pathologic diagnosis of EMPD was 34.7 months (range: 0-74 months). The average time between diagnosis and consultation in the NMSC was 10.3 months (range: 4-74 months). In all but 1 patient with axillary disease, tumours were 
located in the genital region. Mean tumour size was $6.7 \mathrm{~cm}$ (range: $2-15 \mathrm{~cm})$. Of the 15 lesions, $7(47 \%)$ had previously been treated. Topical imiquimod had been used in 71\% (5/7), and surgical excision in $29 \%(2 / 7)$.

After consultation in the NMSC clinic, topical imiquimod was recommended as first-line treatment for 8 lesions in 8 patients. Imiquimod was to be applied 3 times weekly for 3 months, followed by reassessment in clinic. A further course (or courses) of imiquimod was used in the presence of clinical or pathologic evidence of persistent disease. Total imiquimod treatment time averaged 5.5 months (range: $2-12$ months). In 3 of the 8 sites in which imiquimod was used as initial treatment $(38 \%)$, resolution of disease was seen after 4-6 months of use. Residual disease was observed in 5 lesions in 5 patients, 2 of whom continued treatment. Of the other 3 patients, one had not been compliant with treatment and proceeded to have local radiotherapy (54 Gy in 27 fractions). Another requested to stop imiquimod treatment because of painful inflammation and instead underwent local excision with clear margins. The third was lost to follow-up.

Excision at the NMSC clinic was the first-line treatment in 6 patients with 7 lesions. Of those 7 lesions, 2 had previously been treated at another centre. The greatest dimension of the surgical specimens ranged from $1.7 \mathrm{~cm}$ to $7.1 \mathrm{~cm}$ in size. All excisions used wide margins in an attempt to achieve pathologically clear margins, a result that was achieved in only 1 excision. Among the other 6 lesions, gross residual disease in 1 patient was treated with subsequent postoperative radiotherapy (60 Gy in 30 fractions), and microscopic residual disease in 5 cases was treated with imiquimod in 3 cases. In 2 of those cases, no clinical disease was evident after 2 months; and after 3 months of postsurgical imiquimod use, 1 patient had residual disease, but was subsequently lost to follow-up. Of the 2 remaining patients, 1 patient underwent repeat excision with positive margins, and after another 2 months of imiquimod, was rendered disease-free; and 1 patient was lost to follow-up.

After a median follow-up of 2 years, no evidence of disease was seen in $67 \%$ of sites $(10 / 15)$, and residual disease was present or the patient was lost to follow-up in $33 \%$ of sites $(5 / 15)$. Table I summarizes the treatment regimen for each patient.

\section{DISCUSSION}

This report summarizes our experience of 14 patients with 15 EMPD lesions seen in the NMSC clinic at the Odette Cancer Centre. Treatment recommendations included topical imiquimod, surgical excision, and local radiotherapy. Half the patients required more than one treatment modality, highlighting the difficulty in achieving complete eradication of this disease.

In $65 \%$ of all cases, EMPD is found on the vulva in postmenopausal woman ${ }^{5}$. However, the female population is typically referred directly to gynecology at our centre, and so in the NMSC clinic, four times more men than woman with EMPD were seen.

In this series, $21 \%$ of EMPD lesions were associated with other malignancies. In the literature, rates of $31 \%-42 \%$ have been reported. Taking age into account, the increased risk of a second cancer developing in patients with EMPD is $1.7^{3}$. Secondary EMPD (associated with an underlying adenocarcinoma) is less common than primary EMPD, but in patients with associated malignancies, the mortality rate is $46 \%$ higher. In our series, 3 of 14 patients $(21 \%)$ had an associated underlying malignancy (prostate cancer): 2 patients had a prior history of cancer, and 1 patient was diagnosed

TABLE I Overview of patient treatments and outcomes

\begin{tabular}{|c|c|c|c|c|c|c|}
\hline \multirow[t]{2}{*}{ Patient } & \multicolumn{2}{|c|}{ Treatment 1} & \multicolumn{2}{|c|}{ Treatment 2} & \multicolumn{2}{|c|}{ Treatment 3} \\
\hline & Method & Outcome & Method & Outcome & Method & Outcome \\
\hline 1 & Imiquimod & Residual & Radiation & Clear & & \\
\hline 2 & Imiquimod & Residual & Excision & Clear & & \\
\hline 3 & Excision & Residual & Radiation & Clear & & \\
\hline 4 & Imiquimod & Residual & & & & \\
\hline 5 & Excision & Residual & Imiquimod & Clear & & \\
\hline $6^{\mathrm{a}}$ & Excision & Residual & Excision & Residual & Imiquimod & Clear \\
\hline & Excision & Clear & & & & \\
\hline 7 & Excision & Residual & & & & \\
\hline 8 & Excision & Residual & Imiquimod & Residual & & \\
\hline 9 & Imiquimod & Residual & & & & \\
\hline 10 & Excision & Residual & Imiquimod & Clear & & \\
\hline 11 & Imiquimod & Residual & & & & \\
\hline 12 & Imiquimod & Clear & & & & \\
\hline 13 & Imiquimod & Clear & & & & \\
\hline 14 & Imiquimod & Clear & & & & \\
\hline
\end{tabular}

a Patient 6 developed 2 sites of extramammary Paget disease that were separately treated. 
during the post-EMPD diagnosis workup, showing how some malignancies are revealed only after EMPD is identified. Thus, slow diagnosis in patients with EMPD may delay proper treatment of both the lesion and the underlying malignancy, possibly contributing to the higher fatality rate for secondary EMPD.

Extramammary Paget disease is typically an extensive lesion because its subtle onset and nonspecific features often delay the diagnosis by $5-10$ years ${ }^{2}$. At our centre, the average delay from symptom onset to pathologic diagnosis was more than 3 years. In 1 patient, accurate diagnosis was delayed because of a misdiagnosis of Bowen disease - a common error, because Paget and Bowen disease can appear similar clinically ${ }^{2}$. The correct diagnosis can be reached with a careful evaluation of morphology and help from a panel of immunohistochemical markers.

In comparing imiquimod and excision as first-line treatment at the NMSC, imiquimod was more likely than excision to resolve the disease (38\% vs. $14 \%$ respectively). However, in the literature, conventional management is generally surgical excision, although Mohs micrographic surgery is gaining in popularity ${ }^{6}$. Hendi and colleagues ${ }^{7}$ note that when Mohs micrographic surgery is not available, surgical management is also effective when done with wide excision; they specify 5 -cm margins. Lee et al. ${ }^{8}$ found that local control after wide excision is fairly good, with an overall relative 5 -year local control rate of $64.6 \%$. Unfortunately, excision with wide margins can be disfiguring, distorting normal anatomy and leading to difficulties in wound healing. Even though the lesion can be grossly removed, the infiltrating nature of the disease leads to a high incidence of positive margins; frequent follow up is therefore recommended ${ }^{6}$.

Small case studies showed imiquimod to be an effective treatment for EMPD, although complete response may be isolated to more superficial lesions ${ }^{5}$. In agreement with the literature, we found that imiquimod was more successful in treating microscopic disease than gross disease. Imiquimod offers the potential benefit of minimal, if any, long-term morbidity, although acute morbidity includes mild to severe local skin reactions and, in rare cases, systemic flu-like symptoms. Although the skin inflammation will calm in 1-2 weeks, compliance can be problematic for some patients, reducing treatment efficacy.

As an alternative to excision in ineligible patients, radiation is the recommended treatment modality ${ }^{2}$. Radiotherapy spares surgical morbidity and the risk of positive margins leading to treatment with multiple modalities. Moderate doses of radiotherapy (50-60 Gy) over 5-6 weeks are typically required, which in the perineum result in acute moist desquamation and discomfort for the patient. Long-term morbidity can include pigmentation changes, skin atrophy, edema, and ulceration.

Our results suggest that the lower the burden of disease, the more successful imiquimod treatment is (such as in the postoperative setting with microscopic disease). However, because lesion size was not always documented in patient charts, accurately correlating treatment outcome with lesion size is difficult.

\section{CONCLUSIONS}

After reviewing 14 patients at our institute, it would appear that multiple treatment modalities are often required in the management of EMPD. The modality (or sequence of modalities) that most effectively eradicates disease while minimizing treatment toxicity has yet to be determined. Conducting the necessary randomized controlled trials is difficult because of the rarity of the disease; a multi-institutional setting would be required.

\section{CONFLICT OF INTEREST DISCLOSURES}

All authors declare that no financial conflicts of interest exist.

\section{REFERENCES}

1. Crocker HR. Paget's disease affecting the scrotum and the penis. Trans Pathol Soc Lond 1889;40:187-91.

2. Lloyd J, Flanagan AM. Mammary and extramammary Paget's disease. J Clin Pathol 2000;53:742-9.

3. Siesling S, Elferink MA, van Dijck JA, Pierie JP, Blokx WA. Epidemiology and treatment of extramammary Paget disease in the Netherlands. Eur J Surg Oncol 2007;33:951-5.

4. Anolik R, Liang C, Wang N, Rosenman K, Pomeranz M, Joe E. Extramammary Paget disease. Dermatol Online J 2008; 14:15.

5. Kanitakis J. Mammary and extramammary Paget's disease. $J$ Eur Acad Dermatol Venereol 2007;21:581-90.

6. Coldiron BM, Goldsmith BA, Robinson JK. Surgical treatment of extramammary Paget's disease. A report of six cases and a reexamination of Mohs micrographic surgery compared with conventional surgical excision. Cancer 1991;67:933-8.

7. Hendi A, Brodland DG, Zitelli JA. Extramammary Paget's disease: surgical treatment with Mohs micrographic surgery. J Am Acad Dermatol 2004;51:767-73.

8. Lee KY, Roh MR, Chung WG, Chung KY. Comparison of Mohs micrographic surgery and wide excision for extramammary Paget's disease: Korean experience. Dermatol Surg 2009;35:34-40.

Correspondence to: Elizabeth Barnes, Department of Radiation Oncology, Odette Cancer Cancer Centre, 2075 Bayview Avenue, Toronto, Ontario M4N 3M5. E-mail: toni.barnes@sunnybrook.ca

* Radiation Oncology, Sunnybrook Health Sciences Centre, University of Toronto, Toronto, ON.

$\dagger$ Dermatology, Sunnybrook Health Sciences Centre, University of Toronto, Toronto, ON.

$\$ \quad$ Radiation Therapy, Sunnybrook Health Sciences Centre, University of Toronto, Toronto, ON.

$\S \quad$ Plastic Surgery, Sunnybrook Health Sciences Centre, University of Toronto, Toronto, ON. 